\title{
A numerical approach for a class of risk-sharing problems
}

\author{
G. Carlier, A. Lachapelle*
}

January 18, 2010

\begin{abstract}
This paper deals with risk-sharing problems between many agents, each of whom having a strictly concave law invariant utility. In the special case where every agent's utility is given by a concave integral functional of the quantile of her individual endowment, we fully characterize the optimal risk-sharing rules. When there are many agents, these rules cannot be computed analytically. We therefore give a simple convergent algorithm and illustrate it on several examples.
\end{abstract}

Keywords: risk-sharing, comonotonicity, sup-convolution, calculus of variations, numerical approximation.

\section{Introduction}

Risk-sharing problems have their roots in the seminal works of Arrow [1], [2] and Borch [3] in insurance and have received a lot of attention since. Starting from the case of two-agents having preferences given by expected utilities, the theory has been developed in recent years in particular to incorporate more general law invariant preferences such as rank-dependent utilities or monetary risk measures in the financial literature (see Dana [10], Jouini, Schachermayer and Touzi, [14], Carlier and Dana [6], [7], [8] as well as the book by Föllmer and Schied [12] and the references therein).

The fact that comonotonicity is a key property in efficient risk-sharing problems is well-known and has been particularly emphasized by Landsberger and Meilijson in [15] (see [9] for an extension to multivariate risks). For

${ }^{*}$ CEREMADE, UMR CNRS 7534, Université Paris IX Dauphine, Pl. de Lattre de Tassigny, 75775 Paris Cedex 16, FRANCE carlier@ceremade.dauphine.fr, lachapelle@ceremade.dauphine.fr 
suitable law invariant (or quantile-based) and concave utilities, the abstract risk-sharing problem may be brought down to the maximization of some concave functional over the set of comonotone allocations. When utilities are integral functionals of the quantile (which covers the rank-dependent utility case and more generally the so-called rank-linear utility case), this enables one to rewrite the problem as a tractable variational problem subject to a comonotonicity constraint. This reformulation is a key point in the papers mentioned above to analyze and actually solve many instances of the twoagents case.

The theoretical analysis of the many agents-case is not significantly harder than in the two-agents case. However, the analysis of optimality conditions is more involved since many more cases may arise in the many agents case. Typically, optimal risk-sharing rules exhibit ranges of aggregated risk for which a subset of agents is fully insured by the others. In the two-agents case, optimal solutions are combinations of three regimes: agent 1 insures agent 2 , agent 2 insures agent 1 or the solution is interior and thus given by some first-order condition. If there are more agents, there are many more possibilities for the comonotonicity constraint to be binding. We thus claim that the difficulty of the many agents case is in fact a matter of combinatorics and that the search for an efficient computational scheme is therefore natural.

The paper is organized as follows. In section 2, we reformulate a class of risk-sharing problems as tractable variational problems subject to a comonotonicity constraint. More precisely, some notations and preliminaries are given in paragraph 2.1, the two-agents case is then addressed in paragraph 2.2 , we finally show a stability by sup-convolution result in paragraph 2.3 which enables us to reformulate the risk-sharing problem as a comonotonicity constrained variational problem. Optimality conditions for such problems are established in section 3. Finally, a simple and easy to implement algorithm is described in section 4 in which convergence is established and various numerical simulations are presented.

\section{Reformulation of a class of risk-sharing prob- lems}

Let $(\Omega, \mathcal{F}, \mathbb{P})$ be a nonatomic probability space i.e. a probability space such that there is no $A \in \mathcal{F}$ such that $\mathbb{P}(A)>0$ and $\mathbb{P}(B) \in\{0, \mathbb{P}(A)\}$ for every $B \in \mathcal{F}$ with $B \subset A$. The nonatomicity of $(\Omega, \mathcal{F}, \mathbb{P})$ is well-known to be equivalent to the existence of a uniformly distributed random variable on $(\Omega, \mathcal{F}, \mathbb{P})$. Given a random variable $X$ on $(\Omega, \mathcal{F}, \mathbb{P})$, the law of $X$ is denoted 
$\mathcal{L}(X)$. If $X$ and $Y$ are two random variables $X$ on $(\Omega, \mathcal{F}, \mathbb{P})$, we shall denote by $X \sim Y$ the fact that $\mathcal{L}(X)=\mathcal{L}(Y)$.

In the sequel, we will only consider essentially bounded random variables -or risks- on $(\Omega, \mathcal{F}, \mathbb{P})$. We will denote for short by $L_{+}^{\infty}$ the nonnegative cone of $L^{\infty}(\Omega, \mathcal{F}, \mathbb{P})$.

Let $\mathcal{C}$ be the class of utility functions $V: L_{+}^{\infty} \rightarrow \mathbb{R}$ that :

- are strictly concave,

- are monotone, i.e. $V(X) \geq V(Y)$ whenever $(X, Y) \in L_{+}^{\infty} \times L_{+}^{\infty}$ and $X \geq Y$ a.s.,

- law invariant, i.e. $V(X)=V(Y)$ whenever $(X, Y) \in L_{+}^{\infty} \times L_{+}^{\infty}$ and $\mathcal{L}(X)=\mathcal{L}(Y)$

- satisfy the following Fatou property:

$$
V(X) \geq \limsup _{n} V\left(X_{n}\right)
$$

whenever $\left(X_{n}\right)_{n}$ is bounded in $L_{+}^{\infty}$ and $X_{n}$ converges a.s. to $X$.

Given an aggregate risk $X_{0} \in L_{+}^{\infty}$ and $d+1$ agents, $i=1, \ldots, d+1$, each of whom having a utility $V_{i}$ in the class $\mathcal{C}$, the optimal risk-sharing of $X_{0}$ among those agents is determined by solving the sup-convolution problem:

$$
\left(\stackrel{d+1}{\square_{i=1}} V_{i}\right)\left(X_{0}\right):=\sup \left\{\sum_{i=1}^{d+1} V_{i}\left(X_{i}\right), X_{i} \in L_{+}^{\infty}, \sum_{i=1}^{d+1} X_{i}=X_{0}\right\} .
$$

The sup-convolution $\stackrel{d+1}{\square} V_{i}$ is said to be exact if the previous supremum is attained (and then it is attained at a unique point by strict concavity). Our aim is to reformulate the previous risk-sharing problem in a more tractable way using the notion of comonotone allocations. Before we do so, we shall need some preliminaries.

\subsection{Preliminaries}

Let $X$ be a bounded random variable on $(\Omega, \mathcal{F}, \mathbb{P})$ and let $F_{X}(t)=\mathbb{P}(X \leq$ $t$ ), $t \in \mathbb{R}$ denote its distribution function. The generalized inverse of $F_{X}$ (or quantile function of $X$ ) is defined by:

$$
F_{X}^{-1}(t)=\inf \left\{z \in \mathbb{R}: F_{X}(z)>t\right\} \text {, for all } t \in(0,1) .
$$


The random variable $X$ and the quantile function $F_{X}^{-1}$ are then equimeasurable in the sense that for every continuous function $\varphi$ one has:

$$
\mathbb{E}(\varphi(X))=\int_{0}^{1} \varphi\left(F_{X}^{-1}(t)\right) d t
$$

Let us now recall the well known Hardy-Littlewood inequality (see [13], [16], $[4])$.

Proposition 2.1. Let $X$ and $Y$ be in $L_{+}^{\infty}$ then one has

$$
\mathbb{E}(X Y) \leq \int_{0}^{1} F_{X}^{-1}(t) F_{Y}^{-1}(t) d t=\sup _{Z \sim Y} \mathbb{E}(X Z)
$$

and for every concave $u: \mathbb{R} \rightarrow \mathbb{R}$ one has

$$
\mathbb{E}(u(X-Y)) \leq \int_{0}^{1} u\left(F_{X}^{-1}(t)-F_{Y}^{-1}(t)\right) d t
$$

Definition 2.2. Let $X$ and $Y$ be in $L^{\infty}(\Omega, \mathcal{F}, \mathbb{P})$, then $X$ dominates $Y$ in the sense of second order stochastic dominance (which we denote by $X \succeq Y$ ) if $\mathbb{E}(u(X)) \geq \mathbb{E}(u(Y))$ for every concave increasing function $u: \mathbb{R} \rightarrow \mathbb{R}$.

Various characterizations of stochastic dominance are well-known (see [17]) among which:

$$
X \succeq Y \Leftrightarrow \int_{0}^{t} F_{X}^{-1}(t) d t \geq \int_{0}^{t} F_{Y}^{-1}(t) d t, \forall t \in[0,1],
$$

which is equivalent to

$$
\int_{0}^{1} g(t) F_{X}^{-1}(t) d t \leq \int_{0}^{1} g(t) F_{Y}^{-1}(t) d t, \forall g \text { bounded nondecreasing. }
$$

The next statement is originally due to Ryff $[18]$ in the case $(\Omega, \mathcal{F}, \mathbb{P})$ is $[0,1]$ endowed with its Borel field and the Lebesgue measure, we believe it is well-known but give a short proof for the sake of completeness:

Lemma 2.3. Let $X$ and $Y$ be in $L_{+}^{\infty}$, then $X \succeq Y$ if and only if there is a sequence $Z_{n}$ of the form $Z_{n}=\sum_{i=1}^{N_{n}} \lambda_{i}^{n} Y_{i}^{n}$ with $\lambda_{i}^{n} \geq 0, \sum_{i=1}^{N_{n}} \lambda_{i}^{n}=1$ and each $Y_{i}^{n} \sim Y$, such that $Z_{n}$ converges a.s. to $X$.

Proof. Assume first that $Z_{n}$ is of the form mentioned above and $Z_{n}$ converges a.s. to $X$, then for each concave $u$, one has $\mathbb{E}\left(u\left(Z_{n}\right)\right) \geq \mathbb{E}(u(Y))$ and one 
concludes with Lebesgue's dominated convergence theorem (the $Z_{n}$ 's being uniformly bounded).

Conversely, assume $X \succeq Y$ and let us prove that $X \in K$ where $K$ is the closed convex hull in $L^{1}$ of the set $\left\{Z \in L^{1}, Z \sim Y\right\}$ (clearly $K \subset L_{+}^{\infty}$ since $\left.Y \in L_{+}^{\infty}\right)$. If $X \notin K$ it follows from Hahn's-Banach theorem that there exists $P \in L^{\infty}$ and $\varepsilon>0$ such that

$$
\mathbb{E}(P X) \geq \sup _{Z \sim Y} \mathbb{E}(P Z)+\varepsilon .
$$

With Hardy-Littlewood's inequality, this yields

$$
\int_{0}^{1} F_{P}^{-1} F_{X}^{-1} \geq \int_{0}^{1} F_{P}^{-1} F_{Y}^{-1}+\varepsilon
$$

which contradicts $X \succeq Y$ because of inequality (2.3) recalled above. We thus deduce that $X$ is the $L^{1}$ (and thus also a.s. taking a subsequence if necessary) limit of a sequence $Z_{n}$ of the form mentioned in the statement of the lemma.

As a consequence of the previous lemma, we have the following compatibility result which is originally due to Dana [10]:

Lemma 2.4. If a utility function $V$ is in the class $\mathcal{C}$ then it is compatible with second order stochastic dominance that is $V(X) \geq V(Y)$ whenever $(X, Y) \in$ $L_{+}^{\infty} \times L_{+}^{\infty}$ and $X \succeq Y$.

Proof. Assume $X \succeq Y$, using lemma 2.3, $X$ is an a.s. limit of a sequence $Z_{n}$ as in lemma 2.3. Since $V$ is concave and law invariant, $V\left(Z_{n}\right) \geq V(Y)$ and by the Fatou property, we get $V(X) \geq V(Y)$.

A notion that will play an important role in the sequel is that of comonotonicity that we now recall,

Definition 2.5. Let $X_{1}$ and $X_{2}$ be two real-valued random variables on $(\Omega, \mathcal{F}, \mathbb{P})$, then the pair $\left(X_{1}, X_{2}\right)$ is called comonotone if

$$
\left(X_{1}\left(\omega^{\prime}\right)-X_{1}(\omega)\right)\left(X_{2}\left(\omega^{\prime}\right)-X_{2}(\omega)\right) \geq 0 \text { for } \mathbb{P} \otimes \mathbb{P} \text {-a.e. }\left(\omega, \omega^{\prime}\right) \in \Omega^{2} .
$$

A family of random variable $\left(X_{1}, \ldots, X_{d+1}\right)$ on $(\Omega, \mathcal{F}, \mathbb{P})$ is said to be comonotone if $\left(X_{i}, X_{j}\right)$ is comonotone for every $(i, j) \in\{1, \ldots, d+1\}^{2}$.

Roughly speaking $\left(X_{1}, \ldots, X_{d+1}\right)$ is comonotone if all the $X_{i}$ 's evolve in the same direction (or coevolve) and we recall that it is well-known that it is 
equivalent to the fact that each $X_{i}$ can be written as a nondecreasing function of the sum $\sum_{i} X_{i}$ (see for instance [11]). Given $X \in L_{+}^{\infty}$, the set of allocations $\left(X_{1}, \ldots, X_{d+1}\right) \in L_{+}^{\infty}$ that sum to $X$ and are comonotone can then be parametrized by $X_{i}:=x_{i}(X)$ where the $x_{i}$ 's are nondecreasing function that sum to the identity map (on the range of $X$ but this can be extended to the whole line if necessary). Obviously, each map $x_{i}$ is 1-Lipschitz and therefore, by Ascoli's theorem, this parametrization of comonotone allocations with a fixed sum is compact.

Comonotonicity is well-known to be a key property in risk-sharing problems as soon as agents have preferences that are compatible with second order stochastic dominance. Indeed, an important result of Landsberger and Meilijson [15] states that any allocation is dominated (for second order stochastic dominance) by a comonotone one.

\subsection{The two-agents case}

In this paragraph, we study in details the case of two agents $(d=1)$ by a rearrangement argument taken from [9].

Proposition 2.6. Let $V_{1}$ and $V_{2}$ be two utilities in the class $\mathcal{C}$ and $X_{0} \in L_{+}^{\infty}$, there exists a unique $\left(\bar{X}_{1}, \bar{X}_{2}\right) \in L_{+}^{\infty} \times L_{+}^{\infty}$ such that $\bar{X}_{1}+\bar{X}_{2}=X_{0}$ and

$$
V_{1} \square V_{2}\left(X_{0}\right)=V_{1}\left(\bar{X}_{1}\right)+V_{2}\left(\bar{X}_{2}\right) .
$$

Moreover $\left(\bar{X}_{1}, \bar{X}_{2}\right)$ is comonotone hence there exist two nondecreasing functions $\bar{x}_{1}$ and $\bar{x}_{2}:\left[F_{X_{0}}^{-1}(0), F_{X_{0}}^{-1}(1)\right] \rightarrow \mathbb{R}$ such that $\bar{x}_{1}(t)+\bar{x}_{2}(t)=t$ for all $t \in\left[F_{X_{0}}^{-1}(0), F_{X_{0}}^{-1}(1)\right]$ and $\left(\bar{X}_{1}, \bar{X}_{2}\right)=\left(\bar{x}_{1}\left(X_{0}\right), \bar{x}_{2}\left(X_{0}\right)\right)$.

Proof. Let us first suppose that $X_{0}$ has no atom (i.e. $F_{X_{0}}$ is continuous, or equivalently $F_{X_{0}}^{-1}$ increasing). Let $X_{1}$ and $X_{2}$ be in $L_{+}^{\infty}$ such that $X_{1}+X_{2}=$ $X_{0}$, it follows from Jensen's inequality that $\mathbb{E}\left(X_{i} \mid X_{0}\right) \succeq X_{i}$ so that in the sup-convolution, it is enough to maximize over pairs $\left(X_{1}, X_{2}\right)$ that are measurable functions of $X_{0}$. Let $X_{1}=f\left(X_{0}\right)$ and $X_{2}:=X_{0}-f\left(X_{0}\right)$ be such a pair. Since $X_{0}$ is nonatomic there exists a nondecreasing map (or monotone rearrangement) $\tilde{f}$ such that $\tilde{f}\left(X_{0}\right) \sim f\left(X_{0}\right)$ with $0 \leq \tilde{f}\left(X_{0}\right) \leq X_{0}$ (see [19]). Then set $Y_{1}:=\tilde{f}\left(X_{0}\right)$ and $Y_{2}:=X_{0}-\tilde{f}\left(X_{0}\right)$. Since $Y_{1} \sim X_{1}$, $V_{1}\left(Y_{1}\right):=V_{1}\left(X_{1}\right)$, we now claim that $V_{2}\left(Y_{2}\right) \geq V_{2}\left(X_{2}\right)$ and to prove it, thanks to lemma 2.4, it is enough to prove that $Y_{2} \succeq X_{2}$. Let $u$ be a concave function, we have

$$
\mathbb{E}\left(u\left(Y_{2}\right)\right)=\mathbb{E}\left(u\left(X_{0}-\tilde{f}\left(X_{0}\right)\right)=\int_{0}^{1} u\left(F_{X_{0}}^{-1}(t)-\tilde{f}\left(F_{X_{0}}^{-1}(t)\right)\right) d t\right.
$$


but since $\tilde{f}\left(F_{X_{0}}^{-1}\right)=F_{X_{1}}^{-1}$, by proposition 2.1 , we get

$$
\mathbb{E}\left(u\left(X_{2}\right)\right)=\mathbb{E}\left(u\left(X_{0}-X_{1}\right)\right) \leq \int_{0}^{1} u\left(F_{X_{0}}^{-1}(t)-F_{X_{1}}^{-1}(t)\right) d t=\mathbb{E}\left(u\left(Y_{2}\right)\right) .
$$

All this proves that given an admissible pair $\left(X_{1}, X_{2}\right)$ one may find a better one of the form $\left(f\left(X_{0}\right), X_{0}-f\left(X_{0}\right)\right)$ where $f$ is a non decreasing function on $\left[F_{X_{0}}^{-1}, F_{X_{0}}^{-1}\right] \rightarrow \mathbb{R}$ such that $0 \leq f(t) \leq t$ for every $t \in\left[F_{X_{0}}^{-1}(0), F_{X_{0}}^{-1}(1)\right] \rightarrow \mathbb{R}$. In particular, one can find a sequence of such maps $f_{n}$ such that

$$
\lim _{n} V_{1}\left(f_{n}\left(X_{0}\right)\right)+V_{2}\left(X_{0}-f_{n}\left(X_{0}\right)\right)=V_{1} \square V_{2}\left(X_{0}\right) .
$$

Since the functions $f_{n}$ are all nondecreasing and bounded by $\left\|X_{0}\right\|_{\infty}$, it follows from Helly's theorem that there is some (not relabeled) sequence that converges pointwise to some function $f$, hence $\left(f_{n}\left(X_{0}\right), X_{0}-f_{n}\left(X_{0}\right)\right)$ is bounded in $L^{\infty}$ and converges a.s. to $\left(f\left(X_{0}\right), X_{0}-f\left(X_{0}\right)\right)$. The Fatou property guarantees then that

$$
V_{1}\left(f\left(X_{0}\right)\right)+V_{2}\left(X_{0}-f\left(X_{0}\right)\right)=V_{1} \square V_{2}\left(X_{0}\right) .
$$

This proves existence of a maximizer in the sup-convolution problem (that is the sup-convolution is exact at $\left.X_{0}\right)$, uniqueness follows from the strict concavity of $V_{1}$ and $V_{2}$. It remains to show that $\left(f\left(X_{0}\right), X_{0}-f\left(X_{0}\right)\right)$ is comonotone i.e. $X_{0}-f\left(X_{0}\right)$ is nondecreasing in $X_{0}$. To prove this last claim, we apply the same trick as before : let $g$ be nondecreasing such that $0 \leq g(t) \leq t$ and $g\left(X_{0}\right) \sim X_{0}-f\left(X_{0}\right)$, then $X_{0}-g\left(X_{0}\right) \succeq f\left(X_{0}\right)$ so that

$$
V_{1}\left(X_{0}-g\left(X_{0}\right)\right)+V_{2}\left(g\left(X_{0}\right)\right) \geq V_{1}\left(f\left(X_{0}\right)\right)+V_{2}\left(X_{0}-f\left(X_{0}\right)\right)
$$

and therefore $\left(X_{0}-g\left(X_{0}\right), g\left(X_{0}\right)\right)$ is optimal as well and by uniqueness this yields $X_{0}-f\left(X_{0}\right)=g\left(X_{0}\right)$. The supremum is thus uniquely attained at some comonotone pair.

Let us now treat the case where $X_{0}$ is arbitrary in $L_{+}^{\infty}$. A theorem of Ryff ([19]) enables us to write $X_{0}=F_{X_{0}}^{-1}\left(U_{0}\right)$ with $U_{0}$ uniformly distributed. For $n \in \mathbb{N}^{*}$ then define $X_{0}^{n}:=F_{X_{0}}^{-1}\left(U_{0}\right)+n^{-1} U_{0}$, since $X_{0}^{n}$ is nonatomic, we deduce from the previous step that there exists, for every $n \geq 0$, a pair of nondecreasing 1-Lipschitz functions $x_{1}^{n}$ and $x_{2}^{n}$ summing to the identity such that

$$
V_{1} \square V_{2}\left(X_{0}^{n}\right)=V_{1}\left(x_{1}^{n}\left(X_{0}^{n}\right)\right)+V_{2}\left(x_{2}^{n}\left(X_{0}^{n}\right)\right) .
$$

By Ascoli's Theorem, we may extract some converging (and not relabeled) subsequence from $\left(x_{1}^{n}, x_{2}^{n}\right)$ and denote $\left(\bar{x}_{1}, \bar{x}_{2}\right)$ its limit. Now, using the fact 
that $V_{1}$ and $V_{2}$ are monotone and that $X_{0}^{n} \geq X_{0}$, we have, for every $X \in L_{+}^{\infty}$ such that $X \leq X_{0}$,

$$
V_{1}\left(X_{0}-X\right)+V_{2}(X) \leq V_{1}\left(X_{0}^{n}-X\right)+V_{2}(X) \leq V_{1}\left(x_{1}^{n}\left(X_{0}^{n}\right)\right)+V_{2}\left(x_{2}^{n}\left(X_{0}^{n}\right)\right) .
$$

Since $\left(x_{1}^{n}\left(X_{0}^{n}\right), x_{2}^{n}\left(X_{0}^{n}\right)\right)$ is bounded and converges a.s. (and in fact even in $\left.L^{\infty}\right)$ to $\left(\bar{X}_{1}, \bar{X}_{2}\right)=\left(\bar{x}_{1}\left(X_{0}\right), \bar{x}_{2}\left(X_{0}\right)\right)$, we deduce from the Fatou property that the limsup of the right-hand side is less than $V_{1}\left(\bar{x}_{1}\left(X_{0}\right)\right)+V_{2}\left(\bar{x}_{2}\left(X_{0}\right)\right)$. Taking the supremum with respect to $X$ we conclude that the comonotone pair $\left(\bar{x}_{1}\left(X_{0}\right), \bar{x}_{2}\left(X_{0}\right)\right)$ is the unique maximizer in the sup-convolution problem for $X_{0}$.

\subsection{Stability by sup-convolution and existence for $d+1$ agents}

In this section, our aim is to generalize the existence and characterization result of proposition 2.6 to the case of $d+1$ agents. To do so, let us prove the following stability by sup-convolution result.

Proposition 2.7. Let $V_{1}$ and $V_{2}$ be two utilities in the class $\mathcal{C}$, then $V_{1} \square V_{2}$ also belongs to $\mathcal{C}$.

Proof. Let $X_{0}, Y_{0} \in L_{+}^{\infty} \times L_{+}^{\infty}$, then by proposition 2.6, $V_{1} \square V_{2}\left(X_{0}\right)$ and $V_{1} \square V_{2}\left(Y_{0}\right)$ are exact that is, there exist nondecreasing functions $\bar{x}_{1}, \bar{x}_{2}$, and $\bar{y}_{1}, \bar{y}_{2}$ summing to the identity and such that $V_{1} \square V_{2}\left(X_{0}\right)=V_{1}\left(\bar{x}_{1}\left(X_{0}\right)\right)+$ $V_{2}\left(\bar{x}_{2}\left(X_{0}\right)\right)$ and $V_{1} \square V_{2}\left(Y_{0}\right)=V_{1}\left(\bar{y}_{1}\left(Y_{0}\right)\right)+V_{2}\left(\bar{y}_{2}\left(Y_{0}\right)\right)$. Let us prove step by step that $V_{1} \square V_{2}$ belongs to $\mathcal{C}$.

- Strict concavity: let $\lambda \in(0,1)$, and assume $X_{0} \neq Y_{0}$, we thus have $\left(\bar{x}_{1}\left(X_{0}\right), \bar{x}_{2}\left(X_{0}\right)\right) \neq\left(\bar{y}_{1}\left(Y_{0}\right), \bar{y}_{2}\left(Y_{0}\right)\right)$, the strict concavity of $V_{1}$ and $V_{2}$ then yields

$$
\begin{aligned}
V_{1} \square V_{2}\left(\lambda X_{0}+(1-\lambda) Y_{0}\right) \geqslant & V_{1}\left(\lambda \bar{x}_{1}\left(X_{0}\right)+(1-\lambda) \bar{y}_{1}\left(Y_{0}\right)\right) \\
& +V_{2}\left(\lambda \bar{x}_{2}\left(X_{0}\right)+(1-\lambda) \bar{y}_{2}\left(Y_{0}\right)\right) \\
> & \lambda\left[V_{1}\left(\bar{x}_{1}\left(X_{0}\right)\right)+V_{2}\left(\bar{x}_{2}\left(X_{0}\right)\right)\right] \\
& +(1-\lambda)\left[V_{1}\left(\bar{y}_{1}\left(Y_{0}\right)\right)+V_{2}\left(\bar{y}_{2}\left(Y_{0}\right)\right)\right]
\end{aligned}
$$

so that $V_{1} \square V_{2}$ is strictly concave.

- Monotonicity: assume $X_{0} \leqslant Y_{0}$ and let $X \in L_{+}^{\infty}$ be such $X \leqslant X_{0}$, since $V_{1}$ and $V_{2}$ are monotone, we have

$$
V_{1}\left(X_{0}-X\right)+V_{2}(X) \leqslant V_{1}\left(Y_{0}-X\right)+V_{2}(X) \leqslant V_{1} \square V_{2}\left(Y_{0}\right)
$$


taking the supremum with respect to $X$, we deduce that $V_{1} \square V_{2}$ is monotone.

- Law invariance property: assume that $X_{0} \sim Y_{0}$. We then have by law invariance of $V_{1}$ and $V_{2}$

$$
V_{1} \square V_{2}\left(X_{0}\right)=V_{1}\left(\bar{x}_{1}\left(Y_{0}\right)\right)+V_{2}\left(\bar{x}_{2}\left(Y_{0}\right)\right) \leqslant V_{1} \square V_{2}\left(Y_{0}\right),
$$

reversing the role of $X_{0}$ and $Y_{0}$ then yields $V_{1} \square V_{2}\left(X_{0}\right)=V_{1} \square V_{2}\left(Y_{0}\right)$.

- Fatou property: let $\left(X_{n}\right)_{n}$ be a bounded sequence in $L_{+}^{\infty}$ that converges a.s. to a limit $X$. Then for every $n \geq 1$, there exist nondecreasing and 1-Lipschitz nonnegative functions $\bar{x}_{1}^{n}, \bar{x}_{2}^{n}$ such that

$$
V_{1} \square V_{2}\left(X_{n}\right)=V_{1}\left(\bar{x}_{1}^{n}\left(X_{n}\right)\right)+V_{2}\left(\bar{x}_{2}^{n}\left(X_{n}\right)\right),
$$

and, by Ascoli's Theorem, up to a not relabeled subsequence, $\bar{x}_{1}^{n}$ and $\bar{x}_{2}^{n}$ converge uniformly to some $\bar{x}_{1}$ and $\bar{x}_{2}$ so that $\bar{x}_{i}^{n}\left(X_{n}\right)$ converges a.s. to $\bar{x}_{i}(X)$. We thus have

$$
\begin{aligned}
\limsup _{n} V_{1} \square V_{2}\left(X_{n}\right) & \leqslant \limsup _{n} V_{1}\left(\bar{x}_{1}^{n}\left(X_{n}\right)\right)+\limsup _{n} V_{2}\left(\bar{x}_{2}^{n}\left(X_{n}\right)\right) \\
& \leq V_{1}\left(\bar{x}_{1}(X)\right)+V_{2}\left(\bar{x}_{2}(X)\right) \\
& \leq V_{1} \square V_{2}(X)
\end{aligned}
$$

from which we deduce the Fatou property for $V_{1} \square V_{2}$.

Inductively, one immediately deduces from the previous proposition that if $V_{1}, \ldots, V_{d+1}$ all belong to the class $\mathcal{C}$ then so does $\prod_{i=1}^{d+1} V_{i}$.

Theorem 2.8. Let $X_{0} \in L_{+}^{\infty}$ and $V_{1}, \ldots, V_{d+1}$ be in the class $\mathcal{C}$. Then there exists a unique $\bar{X}=\left(\bar{X}_{1}, \ldots, \bar{X}_{d+1}\right) \in\left(L_{+}^{\infty}\right)^{d+1}$ such that $\sum_{i=1}^{d+1} \bar{X}_{i}=X_{0}$ and

$$
{\underset{i=1}{d+1}}_{i=1}^{d} V_{i}\left(X_{0}\right)=\sum_{i=1}^{d+1} V_{i}\left(\bar{X}_{i}\right) .
$$

Moreover $\bar{X}$ is comonotone hence there exist $d+1$ nondecreasing functions $\bar{x}_{i}$ : $\left[F_{X_{0}}^{-1}(0), F_{X_{0}}^{-1}(1)\right] \rightarrow \mathbb{R}$ such that $\sum_{i=1}^{d+1} \bar{x}_{i}(t)=t$ for all $t \in\left[F_{X_{0}}^{-1}(0), F_{X_{0}}^{-1}(1)\right]$ and $\bar{X}=\left(\bar{x}_{1}\left(X_{0}\right), \ldots, \bar{x}_{d+1}\left(X_{0}\right)\right)$. 
Proof. For the sake of simplicity, let us prove the Theorem for $d+1=3$. Introducing the notation $W_{2}=V_{2} \square V_{3}$, the maximization problem reads as:

$$
\sup \left\{V_{1}\left(X_{1}\right)+W_{2}\left(Y_{2}\right) ; X_{1} \in L_{+}^{\infty}, Y_{2} \in L_{+}^{\infty}, X_{1}+Y_{2}=X_{0}\right\} .
$$

By proposition 2.7, we know that $W_{2} \in \mathcal{C}$. Proposition 2.6 then says that there exists a unique solution $\left(\bar{X}_{1}=\bar{x}_{1}\left(X_{0}\right), \bar{Y}_{2}=\bar{y}_{2}\left(X_{0}\right)\right) \in L_{+}^{\infty} \times L_{+}^{\infty}$ such that $\bar{x}_{1}, \bar{y}_{2}$ are nondecreasing, $\bar{x}_{1}\left(X_{0}\right)+\bar{y}_{2}\left(X_{0}\right)=X_{0}$, and

$$
V_{1} \square W_{2}\left(X_{0}\right)=V_{1}\left(\bar{x}_{1}\left(X_{0}\right)\right)+W_{2}\left(\bar{y}_{2}\left(X_{0}\right)\right) .
$$

We then solve the sub-problem

$$
\sup \left\{V_{2}\left(X_{2}\right)+V_{3}\left(X_{3}\right) ; X_{2} \in L_{+}^{\infty}, X_{3} \in L_{+}^{\infty}, X_{2}+X_{3}=\bar{y}_{2}\left(X_{0}\right)\right\} .
$$

By the same arguments, there exists a unique comonotone solution $\left(\bar{X}_{2}=\right.$ $\bar{z}_{2}\left(\bar{y}_{2}\left(X_{0}\right)\right), \bar{X}_{3}=\bar{z}_{3}\left(\bar{y}_{2}\left(X_{0}\right)\right) \in L_{+}^{\infty} \times L_{+}^{\infty}$ satisfying the same properties as in proposition 2.6. Finally, it is easy to see that $\left(\bar{X}_{1}=\bar{x}_{1}\left(X_{0}\right), \bar{X}_{2}=\right.$ $\left.\bar{x}_{2}\left(X_{0}\right), \bar{X}_{3}=\bar{x}_{3}\left(X_{0}\right)\right)$, where $\bar{x}_{2}:=\bar{z}_{2} \circ \bar{y}_{2}$ and $\bar{x}_{3}:=\bar{z}_{3} \circ \bar{y}_{2}$, is the unique solution of the sup-convolution $\stackrel{3}{i=1}_{i}^{3} V_{i}\left(X_{0}\right)$, with the desired properties.

Inductively, one can prove the theorem for every $d$.

Remark 2.9. One may wonder if it is really necessary to proceed inductively to prove the previous result. On the one hand, we believe that proving stability of the class $\mathcal{C}$ by supremal convolution (that is by aggregation) as we did in proposition 2.7 has its own interest. On the other hand, the rearrangement trick we used in the two-agents case does not generalize to more agents.

Remark 2.10. In the previous statements, we have used strict concavity of the elements of $\mathcal{C}$ for uniqueness purpose only. Strict concavity is in fact quite restrictive since it rules out the case of monetary risk measures. We now claim that strict concavity, though convenient because it implies uniqueness, is in fact not necessary to obtain the existence of at least one optimal comonotone solution in the supremal convolution problem. To see this, it is enough to proceed by approximation and use the compactness of comonotone allocations. By the way, in the next section, we will treat numerically the case of concave and non strictly concave utilities by using such strictly concave perturbations.

\subsection{Reformulation}

It follows from the previous results that the risk-sharing problem (2.1) between $d+1$ agents, each of whom has a utility in $\mathcal{C}$, and for aggregate risk 
$X_{0}$, can be simply reformulated as

$$
\sup _{x \in \mathcal{A}} J(x), \text { with } J(x):=\sum_{i=1}^{d} V_{i}\left(x_{i}\left(X_{0}\right)\right)+V_{d+1}\left(X_{0}-\sum_{i=1}^{d} x_{i}\left(X_{0}\right)\right)
$$

where

$$
\begin{gathered}
\mathcal{A}:=\left\{x \in W^{1, \infty}\left([a, b], \mathbb{R}^{d}\right): \dot{x} \in \Delta \text { a.e., } x(a) \in \mathcal{S}\right\}, \\
a:=F_{X_{0}}^{-1}(0), b:=F_{X_{0}}^{-1}(1),
\end{gathered}
$$

and $\Delta$ and $\mathcal{S}$ are the two simplices:

$$
\Delta:=\left\{u \in \mathbb{R}_{+}^{d}, \sum_{i=1}^{d} u_{i} \leq 1\right\}, \mathcal{S}:=\left\{x \in \mathbb{R}_{+}^{d}, \sum_{i=1}^{d} x_{i} \leq a\right\} .
$$

This reformulation is more tractable than the initial one since maximization is now performed over a more concrete set of functions over an interval, namely the convex and compact (for the uniform norm) set $\mathcal{A}$. As we shall see in the next paragraph, the fact that constraints are given by simplices will be very convenient to express necessary conditions.

Of course this reformulation is not very helpful if we keep the previous level of generality on utility functions. This is why we shall now further specify the utilities and restrict them to a subclass for which they have a simple expression in terms of quantiles. From now on, we shall restrict utilities to belong to the class of Rank-Linear Utilities (RLU for short), such utilities are of the form

$$
V_{L}(X):=\int_{0}^{1} L\left(t, F_{X}^{-1}(t)\right) d t .
$$

This class of utilities is already quite large since it contains expected utilities as well as the Rank-Dependent Utilities defined by Choquet expectations. Utilities in this class are obviously law invariant and satisfy the Fatou property as soon as $L$ is continuous (say). RLU have been studied in details in [7], where it is proved in particular that for $V_{L}$ defined by $(2.5)$ (with a $C^{2}$ function $L$ to simplify), the following statements are equivalent:

1. $V_{L}$ is compatible with second order stochastic dominance and monotone,

2. $\partial_{x} L \geq 0, \partial_{x x} L \leq 0$ and $\partial_{t x} L \leq 0$ on $[0,1] \times \mathbb{R}$

3. $V_{L}$ is concave, monotone and $\sigma\left(L^{\infty}(\Omega), L^{1}(\Omega)\right)$ upper semi-continuous. 
Now for a utility of the form (2.5), if $x$ is a nondecreasing function and $X_{0}$ is nonatomic with an increasing distribution function (say), one has

$$
V_{L}\left(X_{0}\right)=\int_{0}^{1} L\left(t, x\left(F_{X_{0}}^{-1}(t)\right) d t=\int_{a}^{b} L\left(F_{X_{0}}(s), x(s)\right) d \mu_{0}(s),\right.
$$

where $\mu_{0}$ is the law of $X_{0}$. In this framework, (2.4) takes the form of a simple variational problem with

$$
J(x):=\int_{a}^{b}\left(\sum_{i=1}^{d} L_{i}\left(F_{X_{0}}(s), x_{i}(s)\right)+L_{d+1}\left(F_{X_{0}}(s), s-\sum_{i=1}^{d} x_{i}(s)\right)\right) d \mu_{0}(s) .
$$

The next paragraphs are precisely devoted to the theoretical and numerical study of such problems (under appropriate regularity and concavity assumptions).

\section{Optimality conditions}

In this section, our aim is to give necessary and sufficient conditions for the problem

$$
\sup _{x \in \mathcal{A}} J(x) \text { with } J(x):=\int_{a}^{b} F(t, x(t)) d t
$$

where we assume that $F \in C\left([a, b] \times \mathbb{R}^{d}, \mathbb{R}\right)$ is such that $F(t,$.$) is strictly$ concave and differentiable for every $t \in[a, b]$ and $\nabla_{x} F$ is continuous in its two arguments.

Under these assumptions, problem (3.1) admits a unique solution $\bar{x}$ that is characterized as follows:

Theorem 3.1. Problem (3.1) admits a unique solution $\bar{x}$ that is characterized by the following:

- $\bar{x} \in \mathcal{A}$,

- for a.e. $t, \dot{\bar{x}}_{i}(t)=0$ for every $i \in\{1, \ldots, d\}$ with $i \notin I(t)$ where

$$
I(t):=\left\{j \in\{1, \ldots, d\}: \bar{p}_{j}(t)=\min _{i=1, \ldots, d} \bar{p}_{i}(t)\right\}
$$

and $\bar{p}$ is the adjoint variable:

$$
\bar{p}(t)=-\int_{t}^{b} \nabla_{x} F(s, \bar{x}(s)) d s .
$$


- $\bar{x}_{i}(a)=0, \forall i \in\{1, \ldots d\}$ with $i \notin I(a)$.

Proof. Existence and uniqueness follow from standard arguments. Let us denote by $\bar{x}$ the solution of (3.1), by concavity of $J$ and convexity of $\mathcal{A}, \bar{x}$ is characterized by the variational inequalities

$$
J^{\prime}(\bar{x}) \cdot(y-\bar{x})=\int_{a}^{b} \nabla_{x} F(t, \bar{x}(t)) \cdot(y(t)-\bar{x}(t)) d t \leq 0, \forall y \in \mathcal{A} .
$$

Now for $y \in \mathcal{A}$, defining $\bar{p}$ by (3.2) and integrating by parts yields

$$
J^{\prime}(\bar{x}) \cdot y=-\int_{a}^{b} \bar{p} \cdot \dot{y}-\bar{p}(a) \cdot y(a)
$$

so that (3.3) becomes

$$
\int_{a}^{b} \bar{p} \cdot \dot{\bar{x}}+\bar{p}(a) \cdot \bar{x}(a)=\inf _{y \in \mathcal{A}}\left\{\int_{a}^{b} \bar{p} \cdot \dot{y}+\bar{p}(a) \cdot y(a)\right\},
$$

now the rightmost member of the previous identity is easy to compute by pointwise minimization over the simplex and is achieved exactly at those $y$ 's in $\mathcal{A}$ such that for a.e. $t \in[a, b], \dot{y}_{i}=0$ as soon as $i \notin I(t)$ and $y_{i}(a)=0$ for $i \notin I(a)$.

In the algorithm of next section, we shall take full advantage of the fact that (3.4) amounts to linear programming over the simplex and is therefore explicit. In particular, for $x \in \mathcal{A}$ and $p$ the associated adjoint variable:

$$
p(t)=-\int_{t}^{b} \nabla_{x} F(s, x(s)) d s,
$$

this characterizes the set of solutions of the linear programming problem

$$
\sup _{y \in \mathcal{A}} J^{\prime}(x) \cdot y
$$

In particular, a solution of (3.6) is given explicitly by:

$$
y(t)=y(a)+\int_{a}^{t} u(s) d s, t \in[a, b]
$$

with

$$
y_{i}(a)= \begin{cases}\frac{a}{\# I_{p}(a)} & \text { if } i \in I_{p}(a) \\ 0 & \text { otherwise }\end{cases}
$$




$$
u_{i}(t)= \begin{cases}\frac{1}{\# I_{p}(t)} & \text { if } i \in I_{p}(t) \\ 0 & \text { otherwise }\end{cases}
$$

and

$$
I_{p}(t):=\left\{j \in\{1, \ldots, d\}: p_{j}(t)=\min _{i=1, \ldots, d} p_{i}(t)\right\} .
$$

For every $x \in \mathcal{A}$, set

$G(x):=\left\{x+\rho(y-x), y \in \operatorname{argmax}_{h \in \mathcal{A}} J^{\prime}(x) \cdot h, \rho \in \operatorname{argmax}_{\lambda \in[0,1]}, J(x+\lambda(y-x))\right\}$.

In the next section, we shall need the following elementary result:

Lemma 3.2. The set valued map $x \in \mathcal{A} \mapsto G(x)$ has a closed graph and $\bar{x}$, the solution of (3.1), is characterized by the condition $\bar{x} \in G(\bar{x})$.

Proof. Let $\left(x_{n}\right)_{n} \in \mathcal{A}$ converge to some $x$ and $z_{n}=x_{n}+\rho_{n}\left(y_{n}-x_{n}\right)$ converge to some $z$ and let us prove that $z \in G(x)$. Taking subsequences if necessary, we may assume that $\rho_{n}$ converges to some $\rho \in[0,1]$ and $y_{n}$ converges uniformly to some $y \in \mathcal{A}$ so that $z=x+\rho(y-x)$. Using the fact that $J$ is of class $C^{1}$ enables us to pass to the limit in

$$
J^{\prime}\left(x_{n}\right) \cdot\left(y_{n}-h\right) \geq 0, \forall h \in \mathcal{A}, J\left(z_{n}\right) \geq J\left(x+\rho\left(y_{n}-x\right)\right), \forall \rho \in[0,1]
$$

and therefore to deduce that $z \in G(x)$.

It is obvious that $\bar{x}$, the solution of (3.1), is such that $\bar{x} \in G(\bar{x})$. Now if $x \in G(x)$ then one has either $\rho=0$ or $x=y$ for some $\rho \in[0,1]$ and some $y \in \mathcal{A}$ as in the definition of $G(x)$. If $x=y$ then $J^{\prime}(x) \cdot h$ is maximized over $\mathcal{A}$ for $h=x$ which is exactly the variational inequality characterizing the solution of (3.1). If $\rho=0$ and $y \neq x$ then $J(x+\lambda(y-x))$ is maximized over $[0,1]$ for $\lambda=0$ so that $J^{\prime}(x) \cdot(y-x) \leq 0$ which again means that $J^{\prime}(x) \cdot h$ is maximized over $\mathcal{A}$ for $h=x$.

\section{Algorithm and numerical results}

\subsection{Algorithm and convergence}

The algorithm we propose to solve (3.1) is a simple optimal step gradient ascent-like method. The fact that the constraints are easy to handle relies again on the fact that linear problems over the simplex are very simple. Our algorithm is defined as follows : 
- start from $x^{0} \in \mathcal{A}$ and define $p^{0}$ as the corresponding adjoint:

$$
p^{0}(t)=-\int_{t}^{b} \nabla_{x} F\left(s, x^{0}(s)\right) d s
$$

- given $\left(x^{k}, p^{k}\right)$ define $y^{k}$ by

$$
y^{k}(t)=y^{k}(a)+\int_{a}^{t} u^{k}(s) d s, t \in[a, b],
$$

with

$$
y_{i}^{k}(a)= \begin{cases}\frac{a}{\# I_{p^{k}}(t)} & \text { if } i \in I_{p^{k}}(a) \\ 0 & \text { otherwise }\end{cases}
$$

and

$$
u_{i}(t)= \begin{cases}\frac{1}{\# I_{p^{k}}(t)} & \text { if } i \in I_{p^{k}}(t) \\ 0 & \text { otherwise. }\end{cases}
$$

Then set

$$
x^{k+1}:=x^{k}+\rho^{k}\left(y^{k}-x^{k}\right)
$$

where

$$
\rho^{k}=\operatorname{argmax}_{\rho \in[0,1]} J\left(x^{k}+\rho\left(y^{k}-x^{k}\right)\right)
$$

which can be computed by simple dichotomy method.

Finally let $p^{k+1}$ be the adjoint state associated to $x^{k+1}$ :

$$
p^{k+1}(t)=-\int_{t}^{b} \nabla_{x} F\left(s, x^{k+1}(s)\right) d s .
$$

The convergence of this simple ascent algorithm is then given by:

Theorem 4.1. The sequence $x^{k}$ generated by the previous algorithm converges to $\bar{x}$, the solution of (3.1) as $k \rightarrow \infty$.

Proof. Since $\mathcal{A}$ is compact (for the uniform topology) and the sequence $\left(x^{k}\right)_{k}$ has values in $\mathcal{A}$, it is enough to show that $\bar{x}$ is the unique cluster point of $\left(x^{k}\right)_{k}$. Let $x$ be such a cluster point, and let $x^{k_{n}}$ be a subsequence converging to $x$, taking a subsequence if necessary we may also assume that $x^{k_{n}+1}$ converges to some $z$. Since $G$ has a closed graph, $z \in G(x)$ i.e. $z$ has of the form $z=$ $x+\rho(y-x)$ with $y$ maximizing $J^{\prime}(x) \cdot y$ over $\mathcal{A}$ and $\rho$ maximizing $J(x+\rho(y-x))$ over $[0,1]$. By construction $J\left(x^{k}\right)$ is nondecreasing, consequently one has $J(x)=J(z)$. Now, if $x \neq \bar{x}$, then by lemma 3.2, one has $x \notin G(x)$ so that $\rho>0$ and $J^{\prime}(x) \cdot(y-x)>0$ which implies that $J(z)=J(x+\rho(y-x))>J(x)$, giving the desired contradiction. This proves that $\bar{x}$ is the only cluster point of $\left(x^{k}\right)_{k}$ and thus that the whole sequence $\left(x^{k}\right)_{k}$ converges to $\bar{x}$. 
Remark 4.2. As already mentioned, it may seem very restrictive to assume strict concavity since it excludes monetary utility functions that are relevant in finance. In fact, as we already explained, strict concavity is by no means essential for existence of an optimal comonotone risk-sharing rule. It is however a crucial assumption for convergence of our algorithm. To deal with problems of the form (3.1) with a concave but not strictly concave $J$ it is enough to consider the perturbed problem

$$
\inf _{x \in \mathcal{A}} J_{\varepsilon}(x) \text { with } J_{\varepsilon}(x):=J(x)+\varepsilon \Phi(x)
$$

where $\varepsilon>0$ is a small perturbation parameter and $\Phi$ is a nice (by this, we mean a smooth integral functional) strictly concave perturbation. Then, our algorithm enables us to compute the solution $\bar{x}_{\varepsilon}$ of the perturbed problem (4.2). Now, it is easy to see that under mild assumptions on the perturbation, $\bar{x}_{\varepsilon}$ converges as $\varepsilon \rightarrow 0^{+}$to the unique maximizer of $\Phi$ among the maximizers of $J$ on $\mathcal{A}$. This perturbation scheme thus naturally selects a particular optimal risk-sharing rule that can be approximated by the algorithm described above.

\subsection{Numerical experiments}

In this final section, we implement the algorithm introduced in section 4.1 and illustrate it on various examples. The numerical results that we obtain confirm the theoretical convergence established in theorem 4.1 and show that our algorithm converges really fast on every example we tested.

More precisely, we first study three particular cases for a uniform distribution of the total risk $X_{0}$ on $[0,1]$. In a fourth example, we compare the solutions for different distributions of $X_{0}$. The last test aims at showing that our numerical procedure is robust for approaching solutions of risk sharing problems with concave but not strictly concave utilities. But before doing so, we would like to mention that the algorithm gives very good results, in particular very quick convergence. Indeed, it is shown on Figure 1-(a) how fast the convergence of the value is. We can also see on Figure 1-(b) that the difference between two successive iterations converges to zero with about the same speed. In all our tests, the algorithm numerically converges in very few iterations (between 5 and 15).

In the following examples, we always take $a=0$ and $b=1$ (which is in fact just a matter of normalization). 

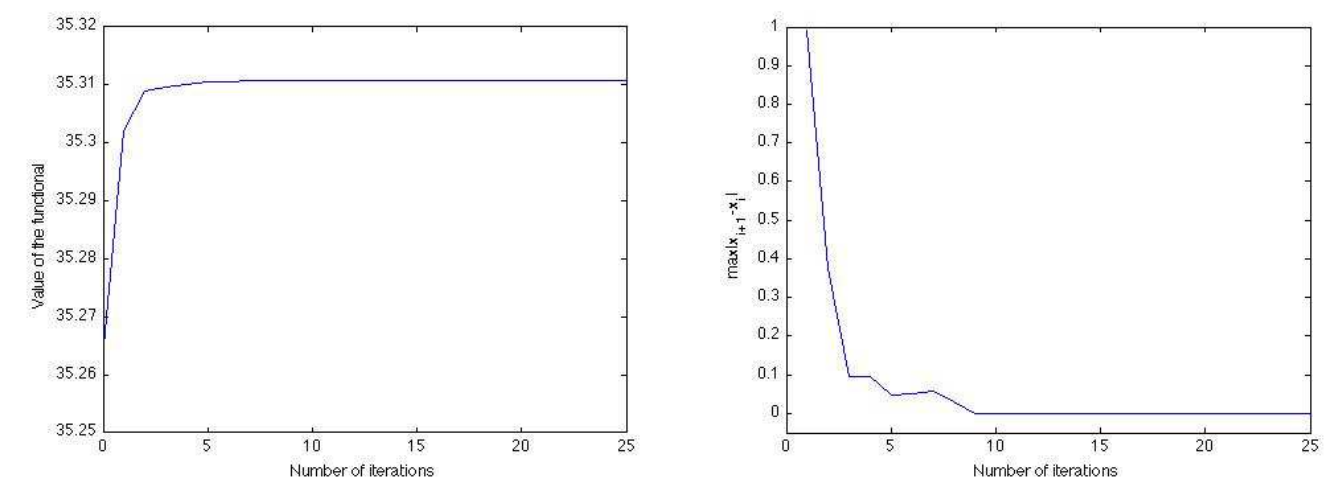

(a) Value of (3.1) at each step of the proce- (b) Convergence of the solution: $\left\|\bar{x}_{i+1}-\bar{x}_{i}\right\|_{\infty}$ dure

Figure 1: Numerical convergence for a 20 agents risk-sharing problem

First case: RDU agents. In the reformulation (2.6), we take functions of the form

$$
L_{i}(t, x)=(1-t)^{\alpha_{i}}(1+x)^{\beta_{i}},
$$

for $i=1, \ldots, d+1$ which corresponds to RDU with power distortion and power utility indices. It is easy to see that for $\alpha_{i} \geq 0$ and $0<\beta_{i}<1$, such functions lead to utilities that belong to the class $\mathcal{C}$. In this first numerical experiment, we look at the case $d+1=5$ and we choose $\alpha:=(2.8,1.6,2.2,1.05,1.3)$ and $\beta:=(0.58,0.52,0.59,0.53,0.57)$. Figure 2 shows the optimal risk-sharing for these data and represents the cumulative sharing in the sense that the size of the risk supported by an agent is the difference between her labeled line (the lines are labeled on the right hand side of the picture) and the nearest sub-line. Looking at Figure 2, one can for instance notice that Agent 4 fully insures the others for any risk with value smaller than 0.34 . Then, for risk values smaller than about 0.7 , Agents 3 and 4 insure the others and finally, for large values of the risk, every agent bears a part of the risk. 


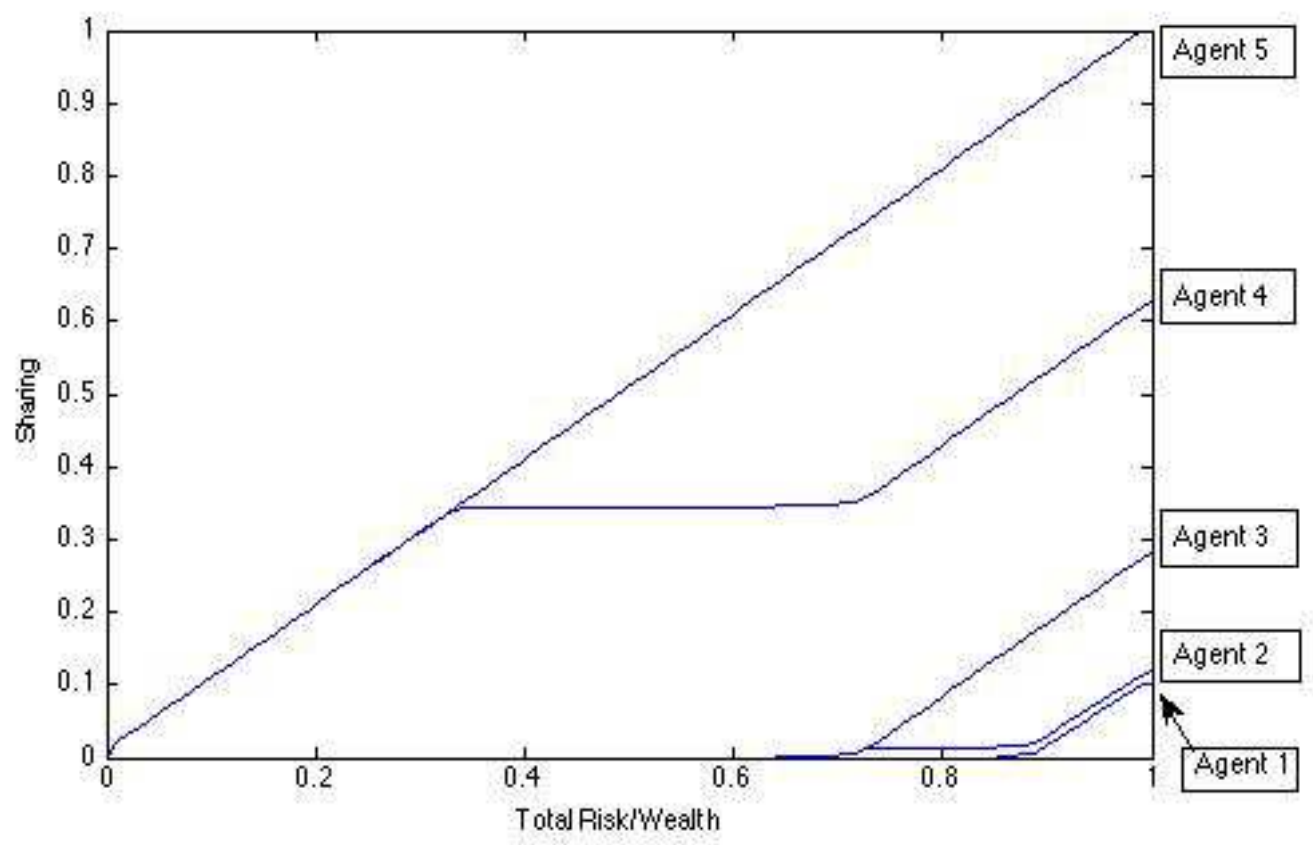

Figure 2: Risk Sharing for 5 agents

Second case: logarithmic RLU. In this example, we make a different choice for the functions $L_{i}$. We take two logarithmic functions $h_{1}(t, x):=$ $\log \left(t^{1 / 2}+x+1\right)$ and $h_{2}(t, x):=\log \left(t^{2 / 3}+x+1\right)$. Note that this RLU example does not correspond to RDU agents. We look at the case of 8 agents, five of them being of type $L_{i}=h_{1}(i=3,4,5,6,7)$ and three of them of type $L_{i}=h_{2}$ $(i=1,2,8)$. The graph of the optimal risk-sharing is represented in Figure 3. The continuous lines correspond to agents of type $h_{1}$ and the discontinuous ones to those of type $h_{2}$. We can see that for risk values between 0 and 0.3 , the three agents of type $h_{2}$ fully insure the others. Numerically, agents of the same type support the same proportion of risk which agrees with the theory.

Third case: mixing RLU and RDU agents. We now turn to a case involving more agents than in the previous ones: $d+1=20$. The functions $L_{i}$ are chosen between $L_{i}(t, x)=(1-t)^{\alpha_{i}}(1+x)^{\beta_{i}}$, for some $\alpha_{i}$ and $\beta_{i}$ satisfying the same assumptions as before, and $L_{i}(t, x)=\log \left(t^{\gamma_{i}}+x+1\right), \gamma_{i} \in(0,1)$. The graph of the solution is represented on Figure 4.

Fourth case: different densities for $X_{0}$. We now aim to see the 


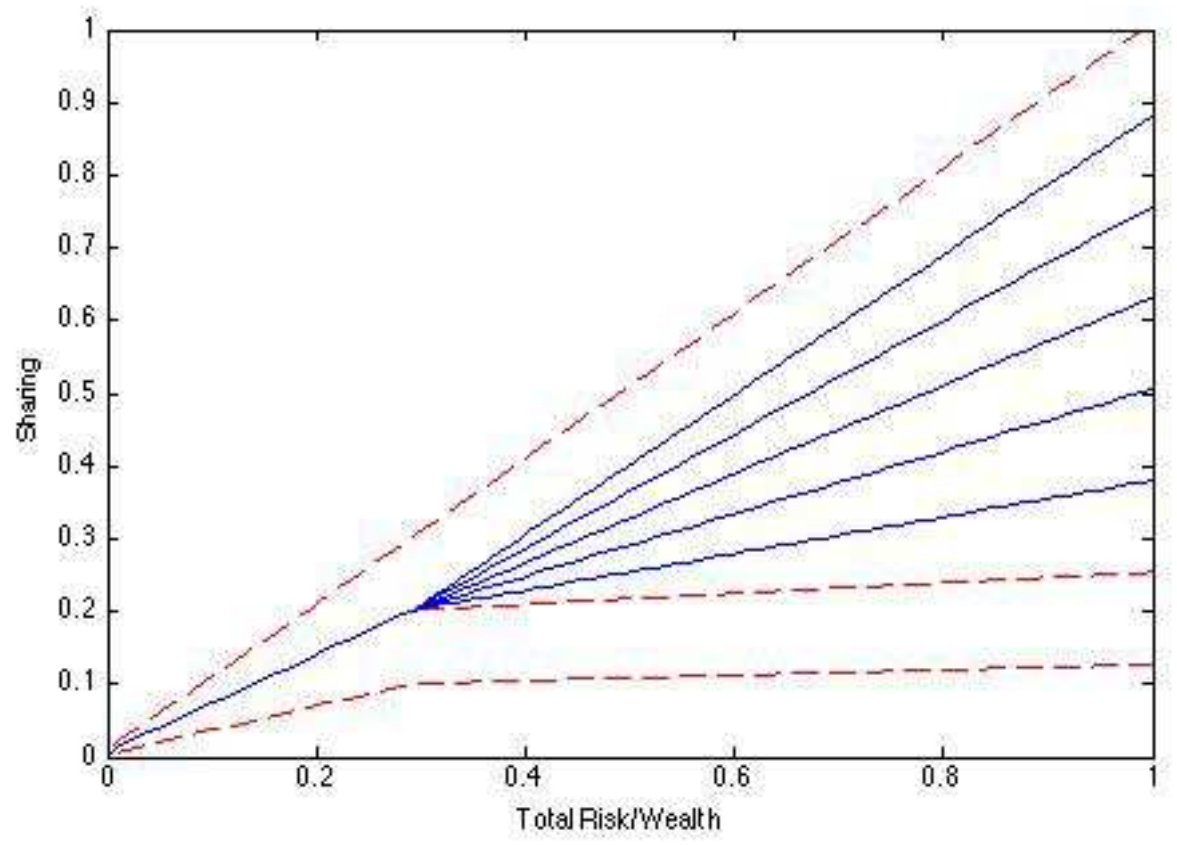

Figure 3: Risk Sharing for 8 agents

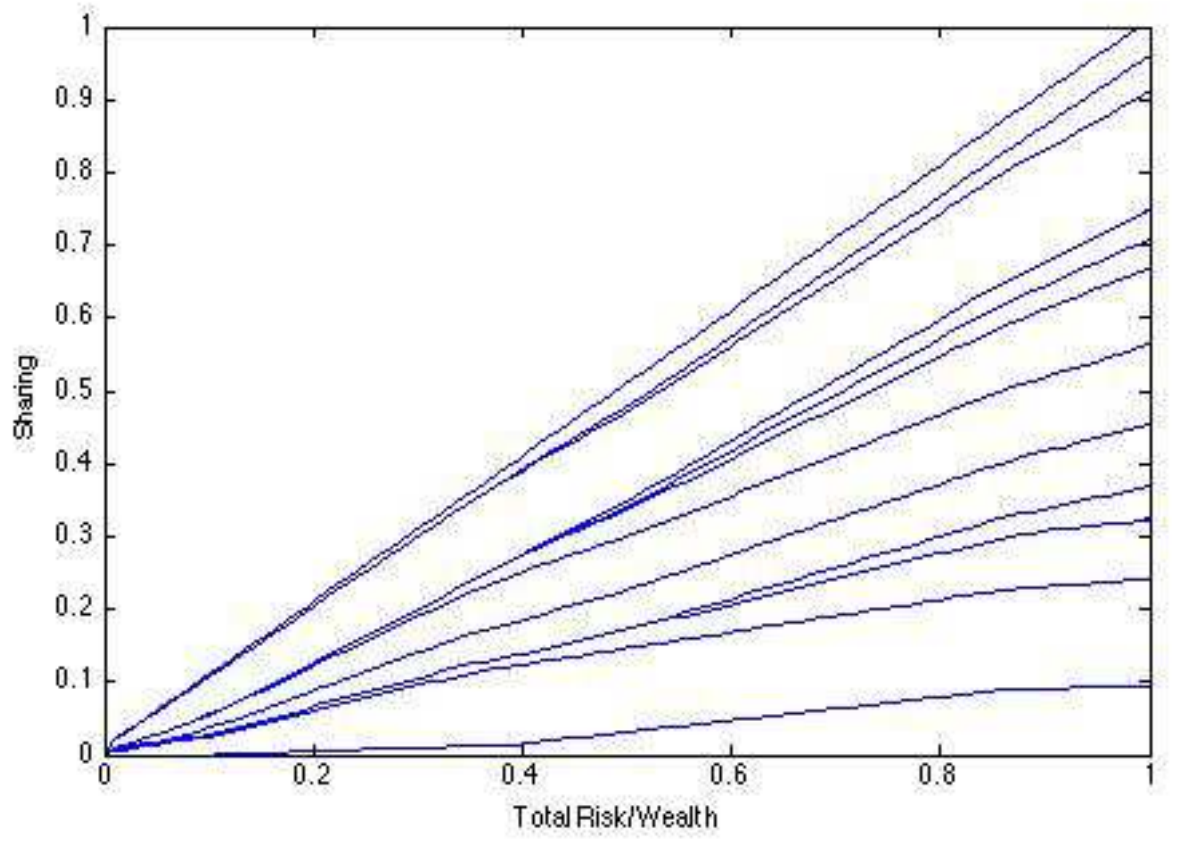

Figure 4: Risk Sharing for 20 agents 


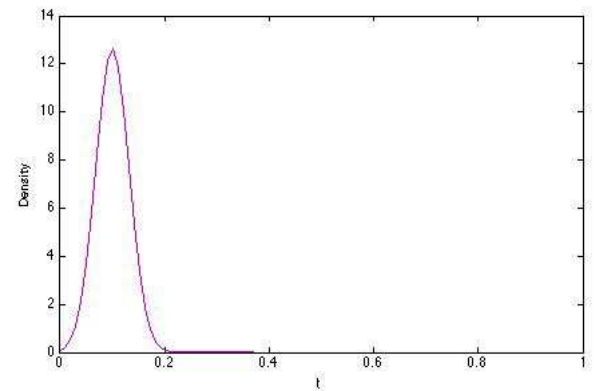

(a) Gaussian density with $\mu=0.1$

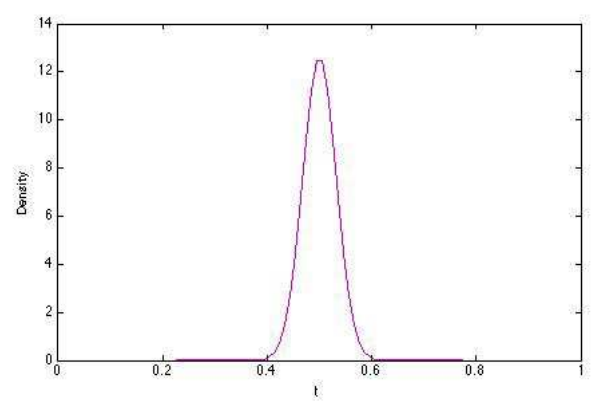

(c) Gaussian density with $\mu=0.5$

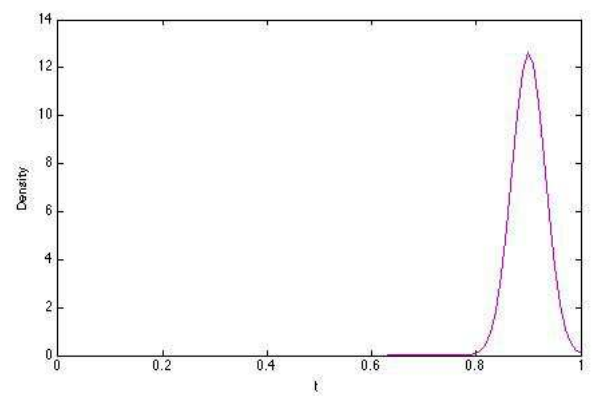

(e) Gaussian density with $\mu=0.9$

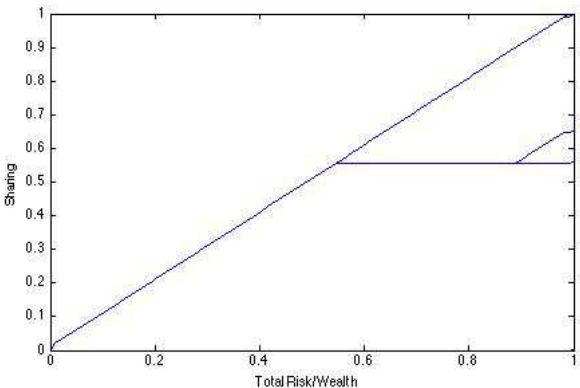

(b) Optimal Risk Sharing for $\mu=0.1$

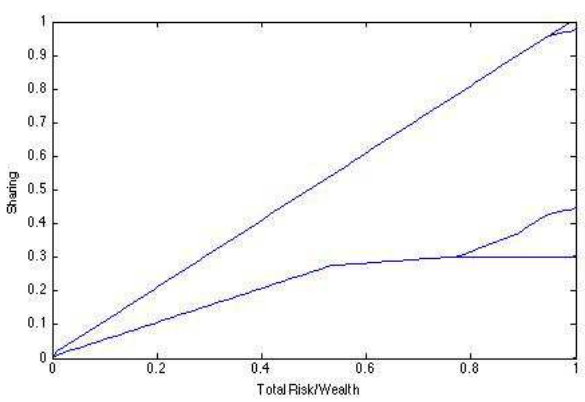

(d) Optimal Risk Sharing for $\mu=0.5$

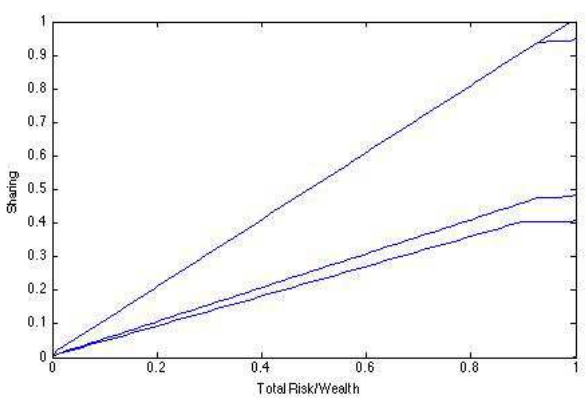

(f) Optimal Risk Sharing for $\mu=0.9$

Figure 5: Plots of the solutions for three averages of a Gaussian distributions of the total risk $X_{0}$.

impact of the law of the aggregate risk $X_{0}$ on the optimal individual risks. To do so, we take $d+1=4$ and functions $L_{i}(t, x)=(1-t)^{\alpha_{i}}(1+x)^{\beta_{i}}$, with the particular choice $\alpha=(1.16,1.89,1.21,2.92)$ and $\beta=(0.52,0.58,0.54,0.59)$. We start with a comparison for three truncated and normalized gaussians with variance $\sigma^{2}=0.01$. More precisely, we plot on Figure 5 the graphs of the density for three different values of the average $\mu=0.1,0.5,0.9$, and 


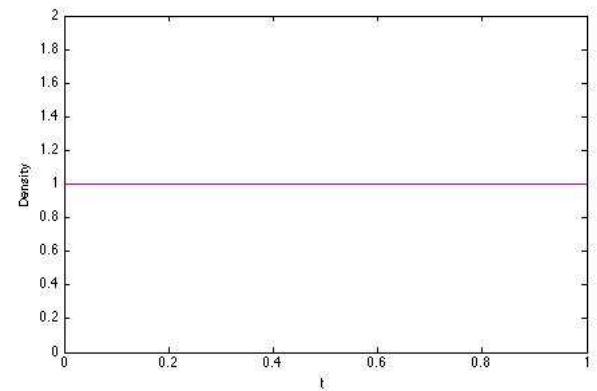

(a) Uniform density

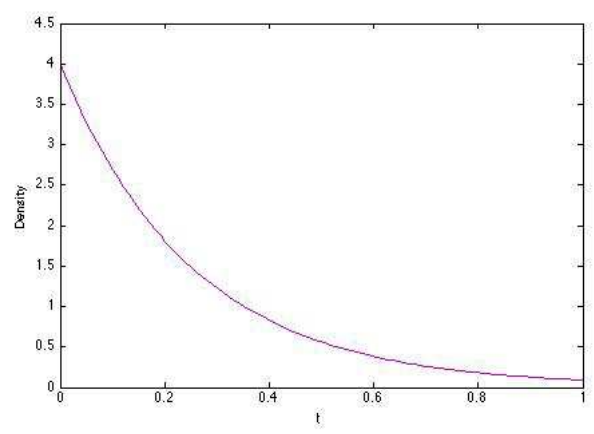

(c) Exponential density with $\lambda=4$

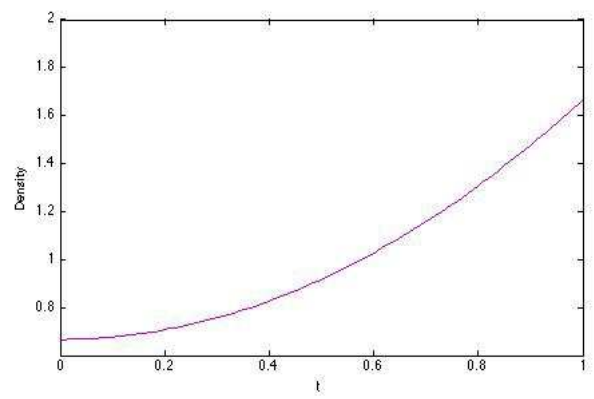

(e) Quadratic density

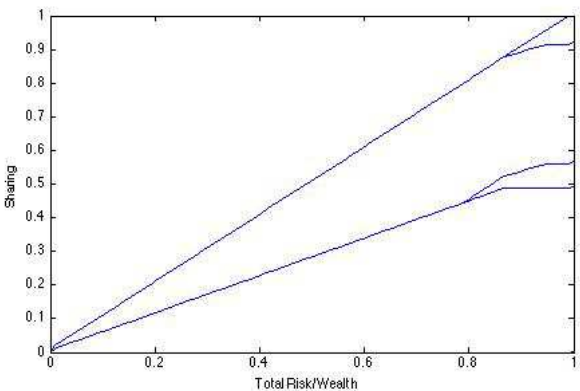

(b) Optimal Risk Sharing for the Uniform density

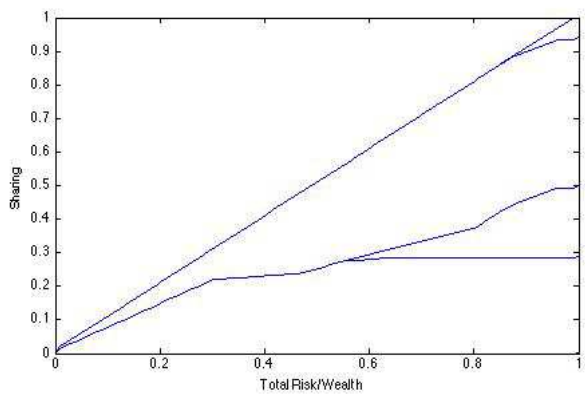

(d) Optimal Risk Sharing for the Exponential density

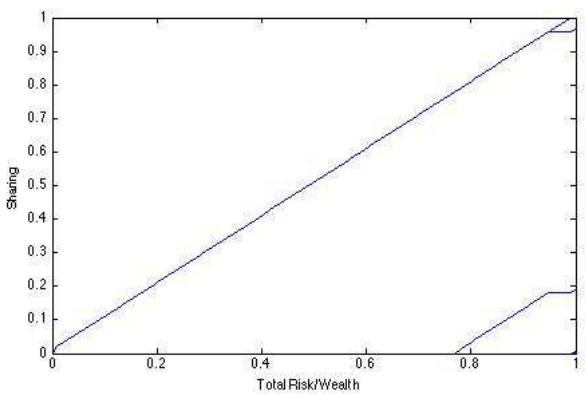

(f) Optimal Risk Sharing for the Quadratic density

Figure 6: Plots of the solutions for three different distributions of the total risk $X_{0}$.

the corresponding solutions. Let us note for instance that Agent 1 fully insures the others for values lower than 0.57 when the average is small (0.1). This phenomenon disappears for larger values of $\mu$. We can also remark that Agent 5 supports a significant proportion of risk only for high values of 
$X_{0}$ and $\mu$. We have drawn on Figure 6 the graphs of three other densities with the corresponding optimal risk-sharing. The densities we study are, for $t \in[0,1]:$

- $(a)-(b): f_{X_{0}}(t)=1$ (Uniform density),

- $(c)-(d): f_{X_{0}}(t)=\lambda e^{-\lambda t}+e^{-\lambda}$ (Exponential-like density),

- $(e)-(f): f_{X_{0}}(t)=t^{2}+\frac{2}{3}$ (Quadratic density, say).

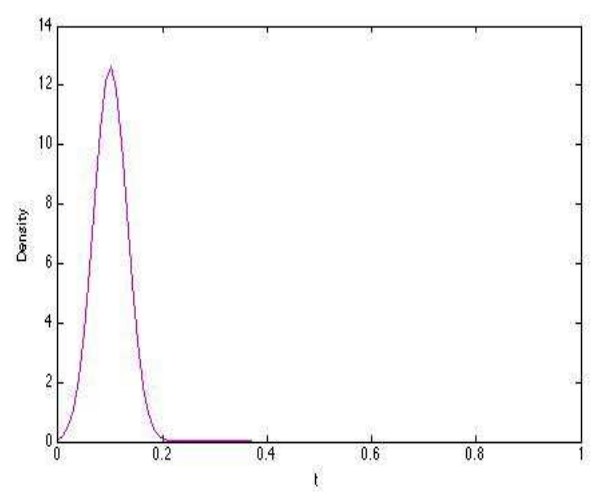

(a) Density of $X_{0}$

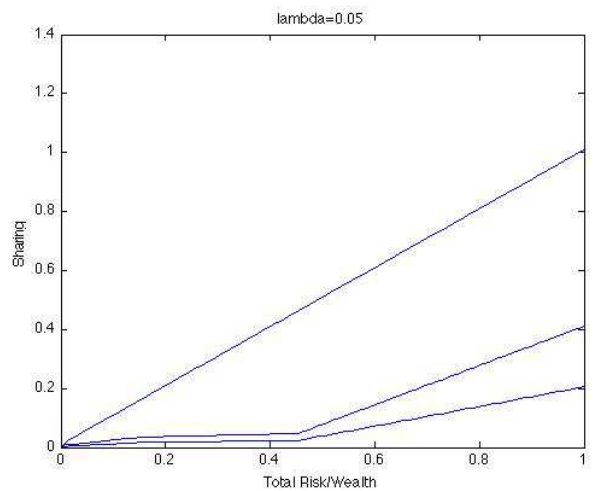

(c) $\varepsilon=0.05$

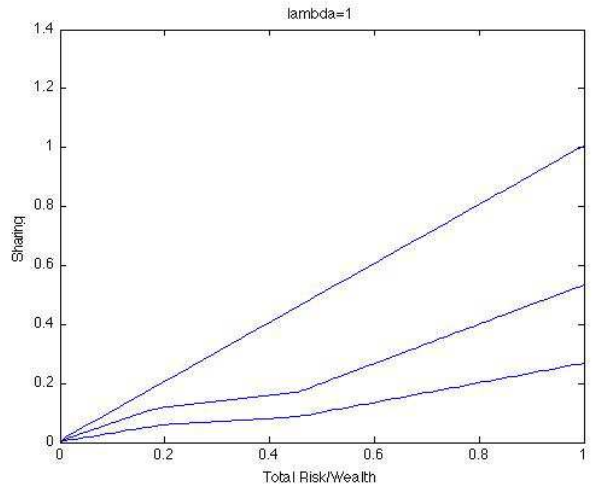

(b) $\varepsilon=1$

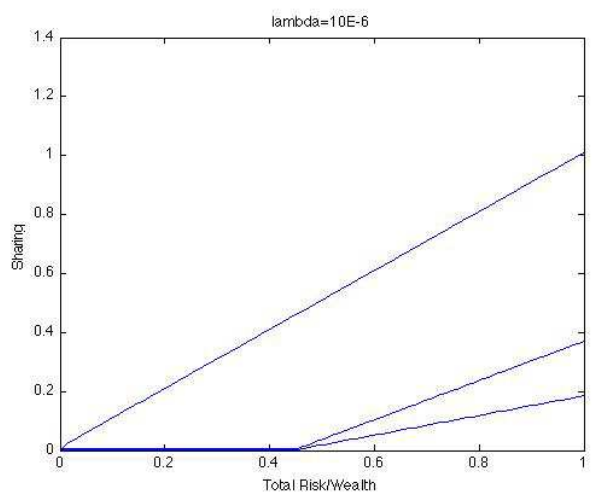

(d) $\varepsilon=10^{-5}$

Figure 7: Approximation of the solution of the problem for a linear utility

Fifth case: non stricly concave utilities. To close this series of numerical tests, we aim to show that our algorithm is actually able to treat concave but not strictly concave utilities. This is an important extension which is motivated by Choquet expectations and monetary risk measures 


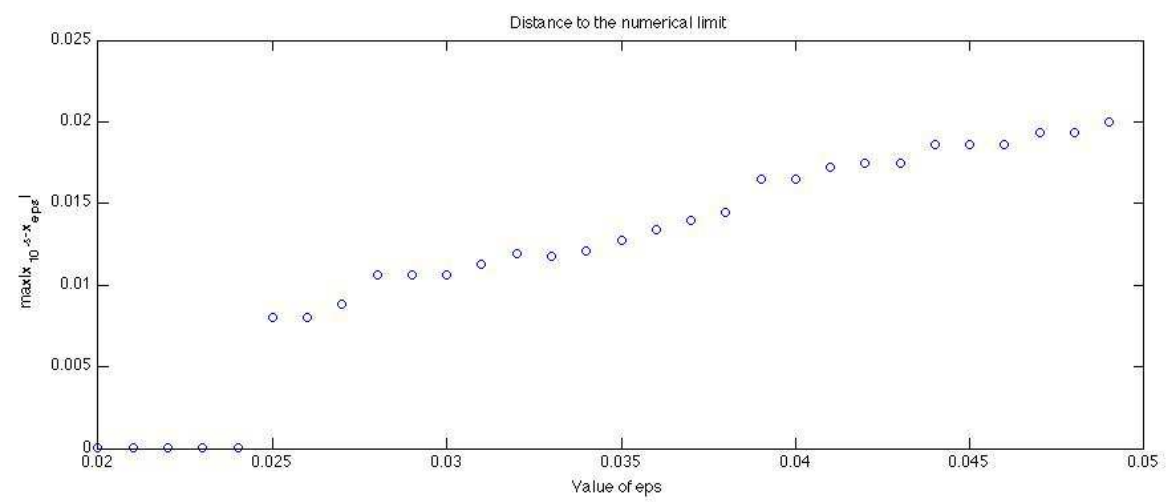

Figure 8: Distance of the approximations to the numerical limit

such as Expected Shortfalls. The idea is to make a small strictly concave perturbation of the utilities as explained in remark 4.2. In this example, we look at a case with 5 agents and a gaussian distribution of the aggregate risk $X_{0}$ (its density is represented on Figure 7-(a)). We then take perturbed utilities of the form

$$
L_{i}^{\varepsilon}(t, x)=\left(1+\varepsilon x^{\beta_{i}}+(1-\varepsilon) x\right)(1-t)^{\alpha_{i}},
$$

with $0<\beta_{i}<1$ and $\alpha_{i}>0$. We then let $\varepsilon \rightarrow 0$ to recover the (linear) Choquet expectation. We show the numerical results for $\varepsilon=1$ (Figure 7(b)),$\varepsilon=0.05$ (Figure 7-(c)) and $\varepsilon=10^{-5}$ (Figure 7-(d)).

We have finally computed the uniform distance between the approximations and the numerical limit. If $\bar{x}_{\varepsilon}$ denotes the numerical solution for a fixed $\varepsilon$, we define this quantity by $\left\|\bar{x}_{10^{-5}}-\bar{x}_{\varepsilon}\right\|_{\infty}$, and we give the results on Figure 8 , for some values of $\varepsilon$ in $[0.02,0.05]$. We therefore observe a numerical convergence.

\section{Acknowledgements}

The authors wish to express their gratitude to Rose-Anne Dana for interesting discussions and helpful suggestions.

\section{References}

[1] K.J. Arrow, Uncertainty and the welfare of medical care, Amer. Econom. Rev. 53 (1963), 941-973.

[2] K.J. Arrow, Optimal insurance and generalized deductibles, Scand. Actuar. J. (1974), 1-42. 
[3] K. Borch, Equilibrium in a reinsurance market, Econometrica, 30 (1962), 424-444.

[4] S. Cambanis, G. Simons and W. Stout Inequalities for $E k(X, Y)$ when the marginals are fixed, Z. Wahrscheinlichkeitstheorie und Verw. Gebiete, 36, (1976), 285-294.

[5] G. Carlier, R.-A. Dana, Core of convex distortions of a probability, Journal of Economic Theory, 113 (2003), 199-222 (2003).

[6] G. Carlier, R.-A. Dana, Law invariant concave utility functions and optimization problems with monotonicity and comonotonicity constraints, Statistics and Decisions, 24 (2006) 127-152.

[7] G. Carlier, R.-A. Dana, Two-Persons Efficient Risk-Sharing and Equilibria for Concave Law-Invariant Utilities, Economic Theory, 36 (2008), 189-223.

[8] G. Carlier, R.-A. Dana, Optimal demand for contingent claims when agents have law invariant utilities, to appear in Mathematical Finance.

[9] G. Carlier, R.-A. Dana, A. Galichon, Pareto efficiency for the concave order and multivariate comonotonicity, preprint available on arxiv.

[10] R.-A Dana, A representation result for concave Schur concave functions, Math. Finance 15 (2005), no. 4, 613-634.

[11] D. Denneberg, Non-additive Measures and Integral, Kluwer Academic Publishers, Holland, 1994.

[12] H. Föllmer, A. Schied, Stochastic finance. An introduction in discrete time, De Gruyter editor, Berlin 2004.

[13] G. H. Hardy, J. E. Littlewood and G. Pòlya, Inequalities, Reprint of the 1952 edition, Cambridge, University Press, Cambridge, 1988.

[14] E. Jouini, W. Schachermayer, N. Touzi, Optimal risk sharing for law invariant monetary utility functions, Math. Finance 18 (2008), 269 292.

[15] M. Landsberger, I.I. Meilijson, Comonotone allocations, Bickel Lehmann dispersion and the Arrow-Pratt measure of risk aversion, Annals of Operation Research 52 (1994) 97-106. 
[16] G.G. Lorentz, An inequality for rearrangements, Amer. Math. Monthly, 60 (1953), 176-179.

[17] M. Rothschild, J. E. Stiglitz,. Increasing Risk, I. A Definition, Journal of Economic Theory 2 (1970) 225-243.

[18] J.V. Ryff, Extreme points of some convex subsets of $L^{1}$, Proc. AMS 116 (1967), 1026-1034.

[19] J.V. Ryff, Measure preserving Transformations and Rearrangements, Journal of Mathematical Analysis and Applications 31 (1970) 449- 458. 